\title{
investigación
}

\section{El Instituto Eduardo Torroja, hoy}

\author{
A. García Meseguer, Dr: Ing. \\ 071-11
}

\section{INTRODUCCION}

El objeto de esta nueva Sección de INFORMES es dar cuenta a los lectores de las investigaciones que se llevan a cabo en el campo de la construcción, no sólo dentro de nuestro Instituto sino también fuera de él. Por ello, en números sucesivos irán desfilando por estas páginas los trabajos en curso de aquellos equipos de investigación de los que tengamos noticia, expuestos en forma rigurosa pero divulgadora: su objeto, sus antecedentes, sus distintas fases, sus logros, sus dificultades. Queremos con ello contribuir a que exista una mayor comunicación entre los técnicos que se ocupan de la realidad cotidiana y los dedicados al estudio en el laboratorio. Ambos pueden ayudarse mutuamente, en especial los segundos a los primeros. Un paso previo es informar y eso es lo que pretendemos con esta nueva Sección.

Ahora bien, como los equipos de investigación del Torroja serán próximos huéspedes de estas páginas - próximos pero no exclusivosel Comité de Redacción de INFORMES ha creído conveniente que comenzásemos por explicar cómo es nuestro Instituto hoy, cuarenta y siete años después de su fundación, treinta y tres años después de aparecer el primer número de INFORMES, veinte años después de la muerte del profesor Eduardo Torroja, fundador del Centro que hoy lleva su nombre. De ahí este artículo.

\section{Instalaciones del Instituto Eduardo Torroja.}

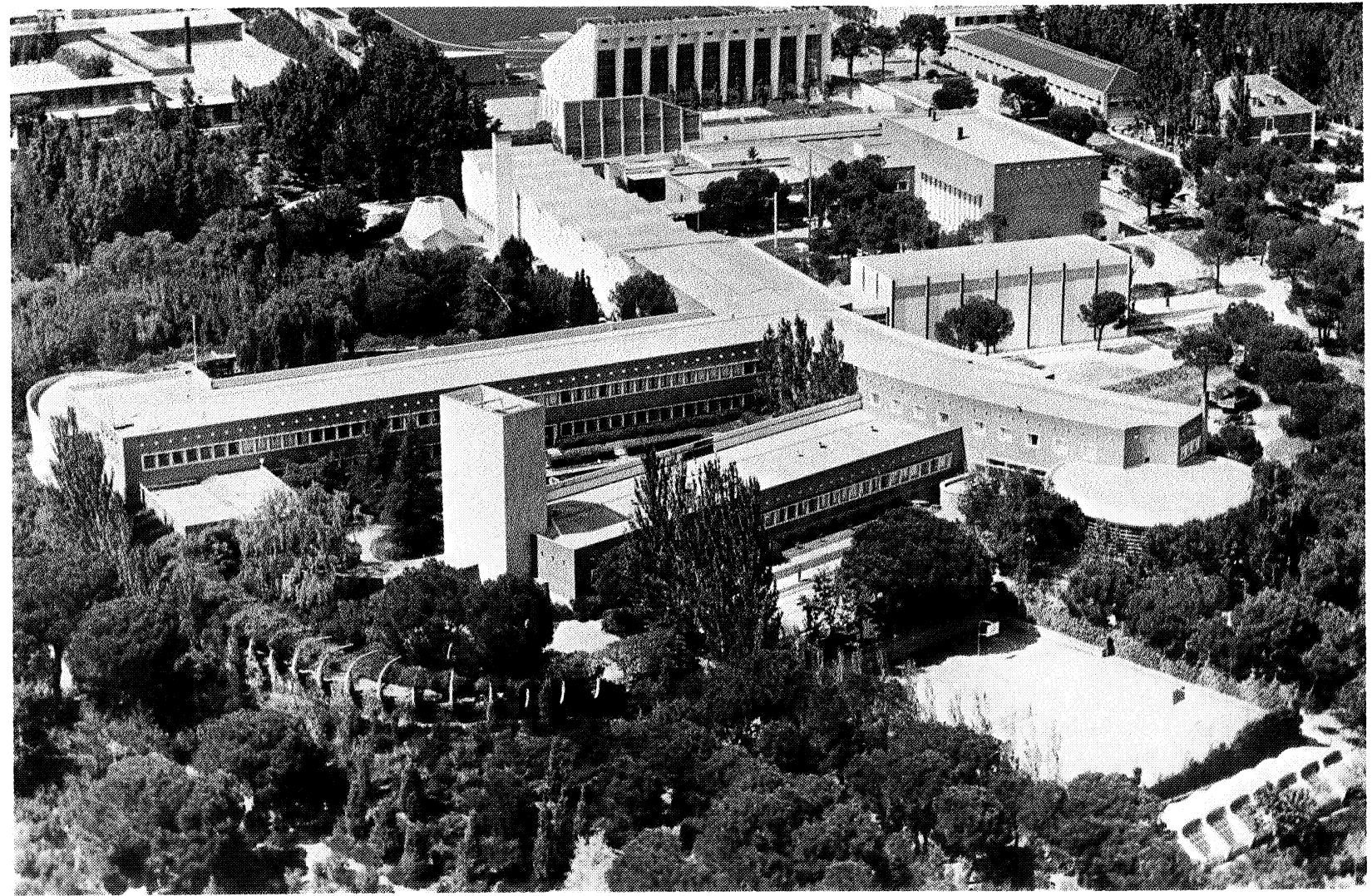




\section{UN POCO DE HISTORIA}

\section{EI IET hasta 1978}

Hasta hace tres años, la estructura del Instituto, su funcionamiento y su modo de gobierno se mantuvieron dentro de una línea que hoy podríamos llamar tradicional. El Centro se estructuraba en Departamentos (de Química, de Materiales, de Estructuras, de Estudios, de Edificación, de Metrología, de Publicaciones, de Asistencia Técnica, etc.) cuyo número y contenido iban variando con el tiempo para adaptarse a las orientaciones de cada época. Las jefaturas de Departamento eran de libre designación del Director quien,
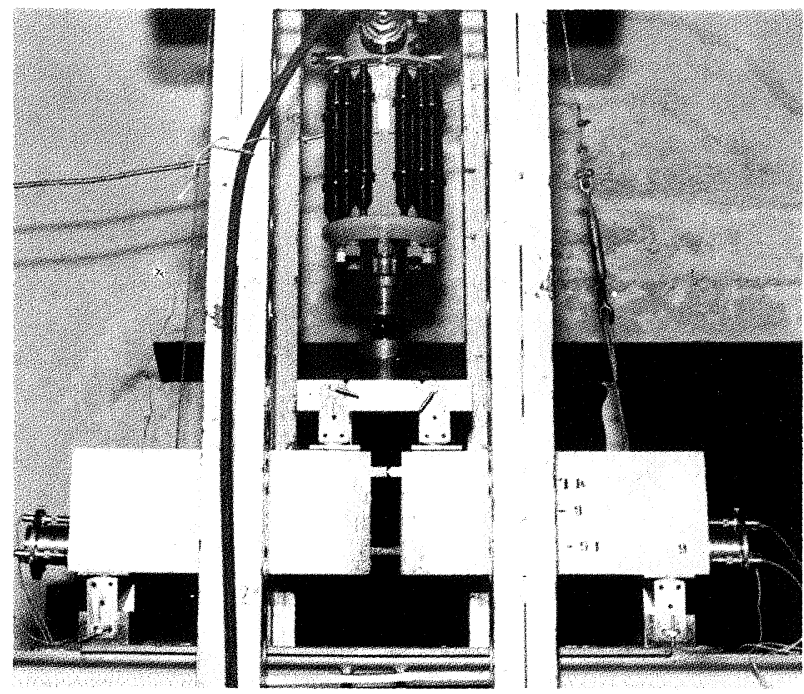

Ensayos de adherencia acero-hormigón de barras corrugadas.

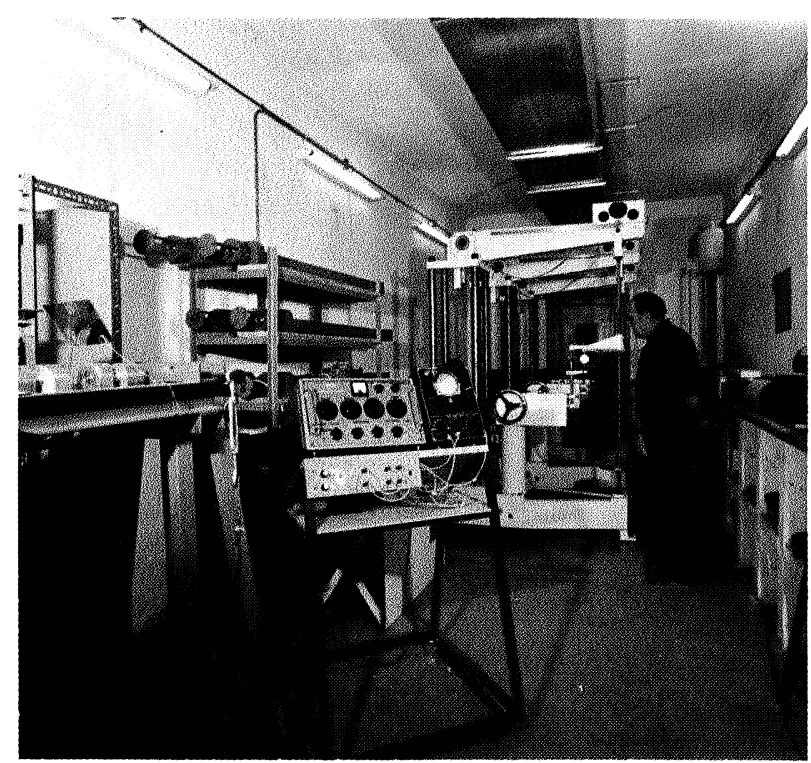

Ensayos de relajación de alambres de pretensado. juntamente con el Subdirector y el Secretario General, aseguraban el gobierno de la casa, asesorados por una Junta de Gobierno que no tenía carácter ejecutivo y cuyos miembros eran también designados por el Director. A su vez, éste era nombrado libremente por la autoridad superior del CSIC (Consejo Superior de Investigaciones Científicas) con duración indefinida.

Los trabajos que se realizaban respondian al contenido del llamado Programa de Trabajos, documento de carácter anual cuya propuesta elaboraba la Dirección en contacto con los Jefes de Departamento. Después, el Consejo Técnico del IETcc sancionaba el Programa y éste se distribuía a todo el personal del Instituto.

\section{EI nuevo Reglamento Orgánico del CSIC}

Este esquema cambia a partir del 23 de enero de 1978 por imperativo de la ley. En efecto, ese día se publica en el B.O.E. el nuevo Reglamento Orgánico (R.O.) del CSIC, el cual establece como nueva forma de trabajo la Planificación por Proyectos de Investigación. Para llevar éstos a cabo, el R.O. crea la figura del Equipo de Investigación, como grupo de personas (en general, de número reducido) que desarrollan un mismo Proyecto y enmarca a todos los integrantes del Instituto en diversas Unidades de Investigación o Unidades de Servicio, que son conjuntos amplios de personas que trabajan en la misma área.

La puesta en marcha de la nueva estructura había de costar bastante tiempo, ya que la filosofía de partida del nuevo R.O. era que todo habría de construirse de forma democrática. Para ello, se definía la figura del Claustro como conjunto del personal científico del Centro y se encomendaba a este corpus la elaboración de una propuesta de reestructuración del Centro, adaptada a los nuevos criterios del CSIC, la cual debía ser aprobada por éste antes de su puesta en marcha definitiva.

\section{La propuesta de reestructuración}

La propuesta de reestructuración se terminó en julio de 1978 y no estuvo exenta de dificultades.

La primera surgió del hecho mismo de la composición del Claustro, ya que en él no estaba representado el personal no científico. Estas personas (un 80 por 100 del personal del Centro) estimaron. injusto que la reestructuración del IETcc se llevase a cabo sin su participación y efectuaron movimientos de protesta comunes a otros Centros del CSIC. El resultado fue que el CSIC creó la figura del Claustro Ampliado (C.A.) cuya composición se obtenía sumando al Claustro un número de representantes del resto del personal igual a la mitad del número de sus miembros, con lo que el C.A. resultaba tener $1 / 3$ de miembros no científicos y $2 / 3$ de miembros científicos ${ }^{(1)}$. Este esquema se aplicó en todos los centros del CSIC. 
El Claustro Ampliado del IETcc era el corpus que debía elaborar la propuesta de reestructuración. A lo largo de sus sesiones (tres meses de intenso trabajo) el C.A. debatió diecisiete informes que le fueron sometidos por otros tantos de sus componentes y elaboró finalmente un documento de 21 páginas en el que se contenía la propuesta de reestructuración ${ }^{(2)}$. Esta propuesta fue remitida al CSIC el 10 de julio de 1978, fecha en la que el C.A. resultó definitivamente disuelto.

\section{Compás de espera}

Casi todos los Institutos del CSIC vieron aprobadas sus propuestas de reestructuración en breve plazo pero éste no fue el caso del Torroja. Cuando en diciembre de 1978 el Consejo Superior de Investigaciones Científicas dictó una normativa detallada para la aplicación del Reglamento Orgánico, en la que se fijaban las etapas a cubrir para poner en práctica la reestructuración, nuestro Instituto no pudo aplicarla por encontrarse todavía pendiente de aprobación su propuesta.

Esta aprobación no llegaría hasta finales de octubre de 1979 y lo fue en términos no idénticos a los contenidos en la propuesta que el Claustro Ampliado había elaborado quince meses antes.

A partir de octubre 79 se inicia un rápido proceso de actividades, que comienza con el encuadramiento voluntario de cada persona en la Unidad de su elección, continúa con las elecciones de los miembros de la Junta de Instituto (cuya composición explicaré más adelante), y culmina con la elección de Director del IETCcc por los miembros de la Junta, en la persona del doctor arquitecto Fernando Aguirre de Iraola, en febrero de 1980.
(1) Dividir a un colectivo como el del IETcc en «cientificos» y «no científicos» es difícil. Cualquiera que sea el criterio de separación (titulación, funciones, categoría administrativa, etc.) siempre se cometen injusticias. Algunas de ellas subsisten todavía.

(2) Personalmente estimo que este documento es bueno y podría ser útil para algún Centro parecido al nuestro.

(3) Elaborado en ocasión de la auditoria emprendida por el CSIC en nuestro Centro, dentro de su programa de evaluación de Institutos.

\section{LA ESTRUCTURA ACTUAL DEL INSTITUTO}

\section{Organigrama del IETcc}

Como se puede apreciar en el organigrama actual del Instituto Torroja ${ }^{(3)}$ el IET consta de tres Unidades de Investigación (llamadas abreviadamente Cemento-Hormigón, Estructuras y Edificación) que encuadran a todo el personal científico de la casa, juntamente con el personal auxiliar cuya actividad fundamental es la investigación; y de tres Unidades de Servicio, que constituyen la infraestructura de apoyo de las anteriores. Al frente de cada Unidad de Investigación hay un Jefe de Unidad elegido de entre sus miembros por el personal con titulación superior que compone la Unidad.

Por otra parte, existen diversos Equipos de Investigación, hasta un total de diez actualmente, cada uno de ellos con un Proyecto concreto que da nombre al equipo.

El número de sus miembros oscila normalmente entre cuatro y siete, de ellos al menos tres titulados. El Jefe de cada Equipo es elegido por los miembros del mismo. Aun cuando todos los componentes de los Equipos están encuadrados en las Unidades de Investigación, los Equipos tienen un funcionamiento autónomo respecto de éstas.

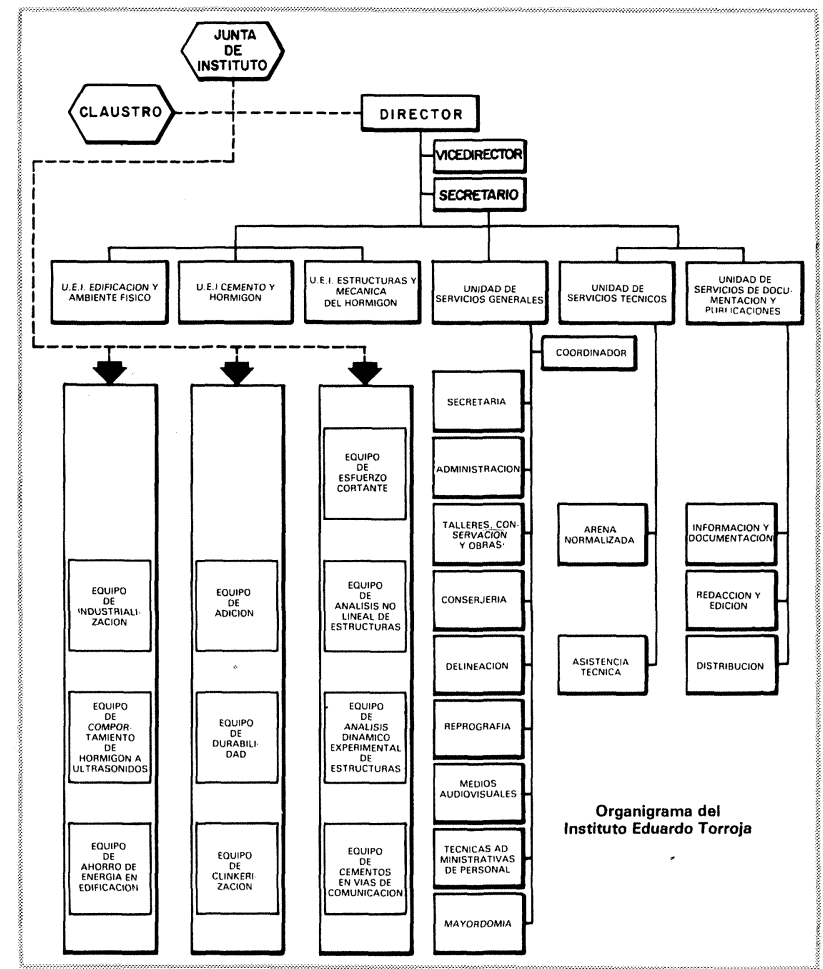


El Equipo de dirección está compuesto por Director, Vicedirector y Secretario. Pero el Gobierno de la casa no reside, realmente, en estas personas, sino en la Junta de Instituto, la cual está compuesta por 24 miembros, a saber:

a) el Director (que actúa de Presidente) y el Vicedirector;

b) los tres Jefes de UEI y los diez Jefes de EI.

c) un representante del personal investigador de carrera;

d) ocho representantes del restante personal, actuando de Secretario el del Centro, con voz pero sin voto.

Por otra parte, el personal investigador, los titulados superiores y los doctores contratados (en la actualidad, 48 personas en total) constituyen el Claustro del IETCcc, cuya función, no ejecutiva, es la de analizar la actividad científica del Centro y proponer las directrices y medidas necesarias para el desarrollo de dicha actividad. Lo preside el Director.

Finalmente, existe un Consejo Técnico cuya función está relacionada con el destino de los fondos que llegan al IETCcc procedentes de las tasas y exacciones de la industria del cemento. En efecto, el R.O. del CSIC otorga a los Consejos Técnicos la misión de proponer los presupuestos de utilización de estos fondos y los programas de investigación y asistencia a que se destinen, así como la de conocer y evaluar los resultados obtenidos.

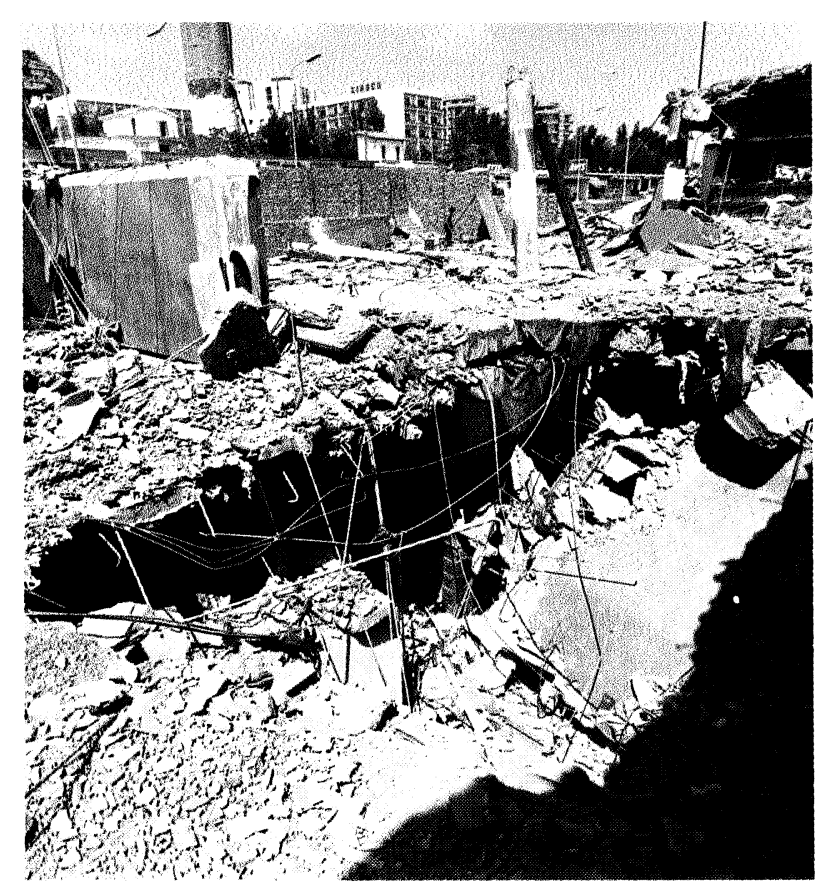

Una aditividad importante: la patología de estructuras.

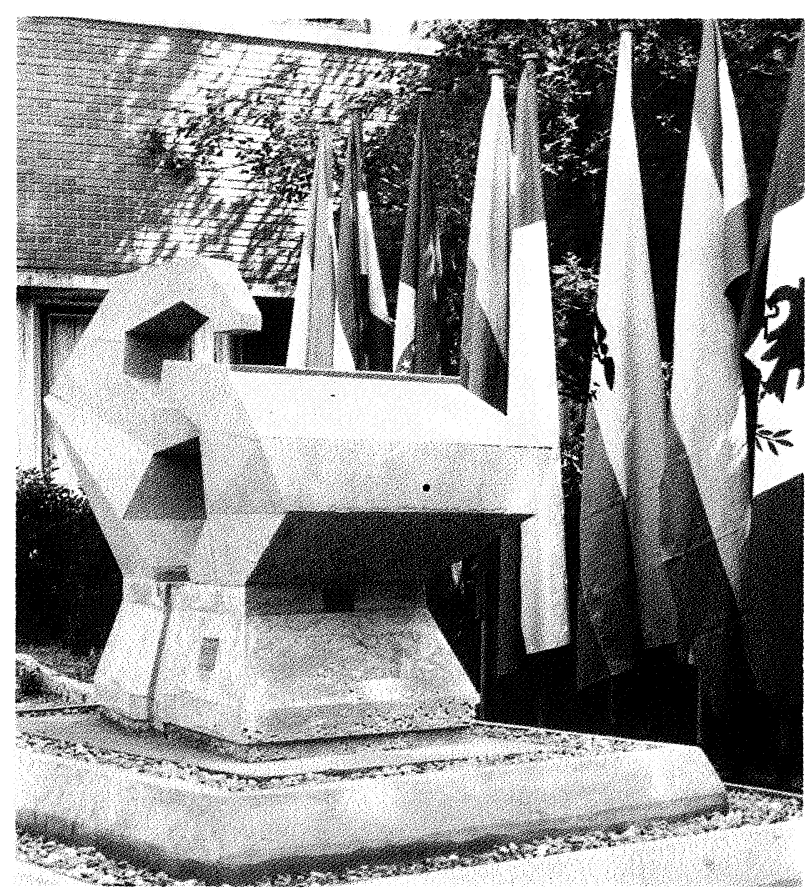

Los cursos CEMCO para profesionales iberoamericanos, una tradición.

\section{Nuestro trabajo actual}

De lo anterior deducirá el lector que el cambio operado en el Instituto es considerable. Un cambio total no puede asimilarse en poco tiempo y por ello no cabe decir que el IETcc se encuentre definitivamente estabilizado en sus nuevos rumbos, aunque el empeño de todos es que así sea en el más breve plazo.

En 1980 la Junta de Instituto intentó preparar un documento en el que se estableciese claramente una definición de funciones del Centro, unos objetivos concretos, unos criterios acerca de los nuevos programas de investigación que debían abordarse y unas directrices para resolver los múltiples problemas de personal que el Instituto tiene, al igual —o aún en mayor grado, debido a su especial carácterque otros Centros del CSIC. Pero la disparidad de opiniones existentes en la Junta impidió que ésta llegase a establecer un documento único sobre los puntos referidos $\mathrm{y}$, al final del año, fueron dos en alternativa los que se enviaron al Presidente del CSIC.

Con buen criterio, el Presidente ha pedido que se haga un esfuerzo de síntesis entre las diversas tendencias, esfuerzo en el que todavía nos encontramos.

A título de ejemplo mencionaré los dos puntos de vista diferentes que existen entre nosotros con respecto a la importancia relativa que debe darse a la investigación teórica y a la cooperación con el exterior. Para unos, el IETcc debería hacer fundamentalmente investigación aplicada $^{(1)}$ con resolución de problemas reales, 
realizando tan sólo aquellas investigaciones básicas que fuesen indispensables para comprender y resolver los problemas técnicos que plantea la investigación aplicada. Para otros, por el contrario, el Instituto debería dedicarse a realizar preferentemente investigación fundamental orientada $^{(1)}$, como paso previo que posibilitaría después una investigación aplicada más avanzada y autónoma. El reflejo práctico de ambas posturas se evidencia en una diferente actitud frente a las peticiones de cooperación que nos llegan del exterior: los primeros tienden a aceptarlas y los segundos a rechazarlas.

La diversidad de criterios no impide que nuestro Instituto siga trabajando. Por estas mismas páginas de la revista irán desfilando los distintos temas de investigación que están en marcha y el lector podrá juzgar acerca de los mismos. Dichos temas no se resumen a los diez que dan nombre a los equipos, sino que abarcan también otros, a cargo de Grupos de Investigación o de personas individuales que, dentro de las Unidades Estructurales de Investigación, trabajan solos o en colaboración con expertos de fuera del IETcc.
Por otra parte, existe un conjunto de temas de cooperación ${ }^{(1)}$ más o menos tradicionales en la casa, que continúan su marcha. Precisamente uno de los aspectos en que la reestructuración emanada del CSIC no coincidió con la propuesta efectuada por el Claustro Ampliado fue éste de la cooperación, ya que, en nuestra propuesta, estas actividades se enmarcaban en una Unidad de Cooperación, mientras que actualmente aparecen dispersas en las diversas Unidades de Investigación y de Servicios.

Un resumen de nuestro trabajo actual aparece en los Cuadros 1,2 y 3 .

Lo que no ha cambiado y sigue guiándonos por encima de las diferencias de opinión, es el lema que preside nuestro Centro: Tecnica plures, opera unica. La técnica es múltiple pero la obra es única.

(1) Ver definiciones en Recuadro.

CUADRO 1.-Actividades de Investigación a cargo de Equipos

\begin{tabular}{|c|c|}
\hline TEMA & OBJETO - AMBITO \\
\hline Clinkerización & $\begin{array}{l}\text { Ahorro de energía en la fabricación del cemento, principalmente mediante sustitución parcial de } \\
\text { productos iniciales y finales del proceso. } \\
\text { Estudios sistemáticos sobre el tema desde hace cinco años. }\end{array}$ \\
\hline Durabilidad & $\begin{array}{l}\text { Comportamiento de hormigones frente a medios agresivos. Predicción del grado de durabilidad de un } \\
\text { cemento frente a un medio agresivo dado. Estudio de protecciones. Banco de datos de obras singulares } \\
\text { con lesiones. Mapa nacional de medios agresivos para la construcción. } \\
\text { Estudios comenzados hace quince años. Se dispone ya de experiencia propia. }\end{array}$ \\
\hline Adiciones & $\begin{array}{l}\text { Estudio, desarrollo y fomento de los cementos con adiciones activas. Incidencia de las adiciones en las } \\
\text { propiedades esenciales del cemento. } \\
\text { Enfocado actualmente al conocimiento de los recursos nacionales de productos activos, con vistas al } \\
\text { ahorro de energía. }\end{array}$ \\
\hline Industrialización & $\begin{array}{l}\text { Desarrollo de una tecnología española para su aplicación en vivienda social y construcciones } \\
\text { escolares. } \\
\text { En fase de reorganización. }\end{array}$ \\
\hline $\begin{array}{l}\text { Ahorro de energía } \\
\text { en edificación }\end{array}$ & $\begin{array}{l}\text { Construcción de un modelo generalizado de cálculo que permita evaluar la idoneidad de una solución } \\
\text { cualquiera (existente o en proyecto) para determinadas condiciones de clima, etc., desde el punto de vista } \\
\text { del confort térmico. } \\
\text { Tema de larga tradición en las tareas del Instituto. }\end{array}$ \\
\hline $\begin{array}{l}\text { Comportamiento del } \\
\text { hormigón a ultrasonidos }\end{array}$ & $\begin{array}{l}\text { Propiedades del hormigón (sano y dañado) frente a impulsos ultrasónicos, con vistas a dictaminar } \\
\text { estructuras sospechosas. } \\
\text { Aplicaciones experimentales en obra desde 1974. Nuevas investigaciones en curso. }\end{array}$ \\
\hline Esfuerzo cortante & $\begin{array}{l}\text { Cierre de un método teórico avanzado para el análisis de elementos rectangulares de hormigón armado } \\
\text { o pretensado sometidos a flexión y cortante. Análisis probabilista del problema. Aplicabilidad de un } \\
\text { nuevo método de elementos finitos. Otros estudios. } \\
\text { Programa subvencionado por la Comisión Asesora del Ministerio de Educación en el periodo 1976-1980. }\end{array}$ \\
\hline $\begin{array}{l}\text { Análisis no lineal y rediseño } \\
\text { de estructuras de hormigón }\end{array}$ & $\begin{array}{l}\text { Estudio teórico y numérico del comportamiento de estructuras de hormigón armado en régimen } \\
\text { no lineal. En particular, edificios sometidos a cargas laterales y pandeo de pórticos de hormigón armado. } \\
\text { En fase activa desde } 1980 \text {. }\end{array}$ \\
\hline $\begin{array}{l}\text { Análisis dinámico } \\
\text { experimental de estructuras }\end{array}$ & $\begin{array}{l}\text { Estudio de la ductilidad y capacidad de absorción de energía de las estructuras de hormigón pretensado, } \\
\text { con particular referencia a las juntas postensadas de elementos prefabricados. Aplicaciones en diseño } \\
\text { sismorresistente. } \\
\text { En fase de reorganización. }\end{array}$ \\
\hline $\begin{array}{l}\text { Cemento en vías de } \\
\text { comunicación }\end{array}$ & $\begin{array}{l}\text { Empleo, con vistas al ahorro energético de materiales locales, materiales de desecho y otros, junto con el } \\
\text { cemento, en vías de comunicación. } \\
\text { Temas parciales finalizarán en } 1983 .\end{array}$ \\
\hline
\end{tabular}


CUADRO 2.-Otras investigaciones a cargo de grupos o personas

TEMA

Concordancia de resultados en ensayos de cemento
OBJETO - AMBITO

Ensayos comparativos entre laboratorios.
Efecto de las corrientes eléctricas en las conducciones metálicas de los edificios

Corrosión de armaduras

Determinar los casos en que una tubería metálica enterrada de conducción de agua puede quedar afectada por fugas de corriente eléctrica.

Estudio del fenómeno en aceros galvanizados. Influencia de los cloruros y de las adiciones. Corrosión de armaduras en cementos aluminosos.

(En colaboración con CENIM y ROCASOLANO)

Hormigones impregnados Estudio de resistencias mecánicas y módulos de elasticidad.
con polímeros

Hormigones con cera Estudio de sus caracteristicas.

Fatigas de gravas-cemento Comportamiento resistente.

\begin{tabular}{ll}
\hline Hormigones porosos & Características drenantes. \\
\hline $\begin{array}{l}\text { Garantía de calidad en } \\
\text { estructuras de hormigón }\end{array}$ & $\begin{array}{l}\text { Establecimiento de un sistema global abarcando todas las fases del proceso constructivo, desde el } \\
\text { planeamiento hasta el uso y mantenimiento. } \\
\text { (En colaboración con expertos europeos del CEB). }\end{array}$ \\
\hline $\begin{array}{l}\text { Construcción a base } \\
\text { de componentes }\end{array}$ & $\begin{array}{l}\text { Estudio de la compatibilidad de componentes y subsistemas de origen industrial en la construcción } \\
\text { de alojamientos. }\end{array}$
\end{tabular}

CUADRO 3.-Principales actividades de Cooperación

\section{TEMA}

Documento de Idoneidad

Técnica

Sellos de Conformidad

Asociaciones nacionales radicadas en el Instituto

Publicaciones

\section{OBJETO - AMBITO}

Dictamen oficial sobre materiales y procesos no tradicionales. Documento con validez automática en varios países europeos, necesario para la exportación. Se realizan unos $15 \mathrm{al}$ año.

Garantía de la calidad estadística de fabricación de materiales. Hay dos en funcionamiento (viguetas para forjados y aceros corrugados para hormigón) y otros en estudio.

ATEP (Asociación Técnica Española del Pretensado).

ATECYR (Asociación Técnica Española de Climatización y Refrigeración).

Revista «Informes de la Construcción» (10 núms./año).

Revista «Hormigón y Acero» (de la ATEP, 4 núms./año).

Revista «Ultimos Avances en Materiales de Construcción» (4 núms./año).

Monografías (entre 4 y 6 al año).

Manuales y Normas.

Libros.

Folletos y otras publicaciones.

Cursos CEMCO

De cinco meses de duración, para ingenieros y arquitectos iberoamericanos. Se imparten cada tres años. Habrá uno en 1982 sobre Patología de la Construcción.

Arena Normalizada

Se fabrica para proporcionar a los laboratorios de ensayo diversos tipos de arena con características perfectamente controladas.

Patología de la Construcción Emisión de dictámenes sobre el estado de estructuras de hormigón y sobre origenes de lesiones en obras de fábrica, cerramientos, cubiertas, etc.

Comisiones nacionales

Colaboración en Comisiones relacionadas con la construcción: de IRANOR, Comisión Permanente del Hormigón, del MOPU, del Ministerio de Industria, Asociación Española para el Control de la Calidad, etc.

Asociaciones Internacionales Colaboración en los trabajos de una veintena de Asociaciones internaciones: CEB, CIB, RILEM, CEM BUREAU, PIARC, AIPC, FIP, etc.

Servicio de Ordenador Expedientes de cálculo de estructuras y otros servicios de cálculo de ingeniería y arquitectura. Abierto al exterior.

Normas Básicas

Colaboración en diversas Normas Básicas del MOPU y otros Ministerios, tales como «Climatización», «Ventilación», «Acústica», «Acciones en la Edificación», «Forjados», etc.

Asistencia Técnica

Ensayos de laboratorio y emisión de dictámenes sobre materiales, componentes, elementos e instalaciones de la construcción. Se tiende a reducir estas actividades, especialmente las de carácter rutinario. 


\section{RECUADRO}

\begin{tabular}{|c|c|}
\hline C.S.I.C. & Consejo Superior de Investigaciones Científicas, al que pertenece el Instituto Eduardo Torroja. \\
\hline Proyecto de investigación: & $\begin{array}{l}\text { Trabajo a desarrollar por un equipo, con una finalidad concreta y aplicando unos medios } \\
\text { determinados, con plazo definido de realización. }\end{array}$ \\
\hline Equipo de investigación (EI): & $\begin{array}{l}\text { Grupo reducido de personas (normalmente entre cuatro y siete, al menos tres de ellas } \\
\text { tituladas) que desarrollan un proyecto de investigación. Su jefe forma parte de la Junta. }\end{array}$ \\
\hline Grupo de investigación: & $\begin{array}{l}\text { Persona o grupo reducido de personas que desarrollan un proyecto de investigación. No } \\
\text { tiene representación en la Junta. }\end{array}$ \\
\hline $\begin{array}{l}\text { Unidad Estructural de } \\
\text { Investigación (UEI): }\end{array}$ & $\begin{array}{l}\text { Conjunto amplio de personas cuya misión principal es el mantenimiento en vanguardia } \\
\text { de la disciplina que cultivan y la participación en equipos de investigación. Su jefe forma } \\
\text { parte de la Junta. }\end{array}$ \\
\hline Claustro: & $\begin{array}{l}\text { Conjunto del personal investigador, titulados superiores y doctores contratados del Instituto, } \\
\text { cuya misión es analizar la actividad científica del Centro y proponer las directrices y medidas } \\
\text { necesarias para el desarrollo de dicha actividad. Está compuesto actualmente por } 48 \text { miembros. }\end{array}$ \\
\hline Junta: & Organo ejecutivo del Instituto. Está compuesto actualmente por 24 miembros. \\
\hline Investigación fundamental: & $\begin{array}{l}\text { Trabajo experimental o teórico dirigido primordialmente a la adquísición de nuevos } \\
\text { conocimientos sobre los fundamentos subyacentes a los fenómenos y hechos observados, } \\
\text { sin ningún uso o aplicación particular a la vista. }\end{array}$ \\
\hline $\begin{array}{l}\text { Investigación fundamental } \\
\text { libre: }\end{array}$ & $\begin{array}{l}\text { La emprendida por los cientificos hacia el logro de sus propias metas, organizando su } \\
\text { propio trabajo. }\end{array}$ \\
\hline $\begin{array}{l}\text { Investigación fundamental } \\
\text { orientada: }\end{array}$ & $\begin{array}{l}\text { La emprendida por los científicos, orientada y dirigida hacia amplios campos de interés } \\
\text { general. }\end{array}$ \\
\hline Investigación aplicada: & $\begin{array}{l}\text { Investigación original emprendida en orden a adquirir nuevos conocimientos y dirigida } \\
\text { primordialmente hacia un propósito y objetivo práctico especifico. }\end{array}$ \\
\hline Cooperación: & $\begin{array}{l}\text { Conjunto de actividades cuyo objetivo primordial no es la adquisición de nuevos conocimientos } \\
\text { sino la aplicación, difusión o intercambio de los conocimientos existentes. }\end{array}$ \\
\hline
\end{tabular}

